\title{
Comparison of VOC Emissions from Natural Wood and Heat Treated Wood
}

\section{Usporedba emisije hlapljivih organskih spojeva iz prirodnog drva i toplinski obrađenog drva}

\author{
Original scientific paper • Izvorni znanstveni rad \\ Received-prispjelo: 13. 2. 2017. \\ Accepted-prihvaćeno: 27. 11. 2018. \\ UDK: $630 * 845.54$ \\ doi:10.5552/drind.2018.1708
}

\begin{abstract}
This paper describes the emissions of Volatile Organic Compounds (VOC) emitted by solid Spruce (Picea abies) and Poplar (Populus alba) wood treated at $200^{\circ} \mathrm{C}$ and $180^{\circ} \mathrm{C}$. The emissions of VOC from heat treated wood and air-dried (natural) wood were compared with GC-MS analysis. The focus was on the influence of temperature, on the quality and quantity of volatile organic compounds, especially on the amount of emitted phenol and furfural. Furfural and phenol are typical chemicals produced as a result of thermal degradation of wood components. The emission was analyzed as a function of time after heat-treatment. The influence of the finishing with waterborne lacquer on VOC emission was also investigated.

The results of this research have shown that heat treated wood emitted more VOC-components of furfural and phenol than untreated wood (natural wood). The amount of emitted VOC declines with increasing the time between wood modification and measurement of VOC emissions emitted by tested samples. Heat treated wood finished by waterborne lacquer emitted a very high concentration of Buthoxy-ethanol. The finished surface does not decrease the amount of emissions escaping from heat-treated spruce and poplar wood.
\end{abstract}

Keywords: VOC emissions, spruce, poplar, heat-treated wood, gas chromatography

SAŽETAK • U radu su predstavljeni rezultati istraživanja emisije hlapljivih organskih spojeva (VOC) iz cjelovitog drva smreke (Picea abies) i drva topole (Populus alba), toplinski obrađenoga pri 180 i $200{ }^{\circ} \mathrm{C}$. Emisije VOC-a iz toplinski obrađenog drva i iz prosušenoga neobrađenog (prirodnog) drva uspoređene su primjenom GC-MS analize. Naglasak istraživanja bio je na utjecaju temperature na kvalitetu i količinu hlapljivih organskih spojeva, posebice na količinu emitiranog fenola i furfurala. Furfural i fenol tipični su kemijski spojevi koji nastaju toplinskom degradacijom drvnih komponenata. Emisija VOC-a analizirana je u ovisnosti o vremenu nakon toplinske obrade. Istraživan je i utjecaj završne obrade drva premazima na bazi vode na emisiju VOC-a.

Rezultati istraživanja pokazali su da toplinski obrađeno drvo emitira više VOC komponenata furfurala i fenola nego neobrađeno (prirodno) drvo. Količina emitiranog VOC-a smanjuje se s porastom vremenskog razmaka između toplinske obrade drvnih uzoraka i mjerenja emisije VOC-a. Toplinski obrađeno drvo koje je završno obrađeno

\footnotetext{
Author is research assistant at Faculty of Forestry and Wood Technology, Mendel University in Brno, Czech Republic.

Autor je znanstveni asistent Fakulteta šumarstva i drvne tehnologije, Mendelovo sveučilište u Brnu, Republika Češka.
} 
vodenim lakom emitiralo je vrlo visoku koncentraciju butoksietanola. Završna obrada toplinski obrađenog drva vodenim lakom nije pridonijela smanjenju emisije VOC-a iz toplinski obrađenog drva smreke i topole.

Ključne riječi: emisije hlapljivih organskih spojeva, smreka, topola, toplinski obrađeno drvo, plinska kromatografija

\section{INTRODUCTION 1. UVOD}

Heat-treatment of timber is used to modify the properties of wood to resist dimensional changes in different humidity (Westin et al., 2001), to achieve better heat insulation, improved decay and weather resistance, reduced deformations due to changes of equilibrium moisture content and new shades of colour as an alternative to tropical hardwood.

The heat treatment process involves exposing wood to elevated temperatures from 160 to $260{ }^{\circ} \mathrm{C}$ (Militz, 2002). The temperature and duration of heat treatment generally vary from 180 to $280{ }^{\circ} \mathrm{C}$ and $15 \mathrm{~min}$. to $24 \mathrm{~h}$ depending on the process, wood species, sample size, moisture content of the sample and the desired mechanical properties, resistance to biological attack and dimensional stability of the final product (Militz, 2002; Kamdem et al., 2002; Sanderman and Augistin, 1964).

Heat-treated wood is an eco-friendly alternative to impregnated wood materials, and heat treated wood can be used for garden, kitchen and sauna furniture, cladding on wooden buildings, bathroom cabinets, floor material, musical instruments, ceilings, inner and outer bricks, doors and window joinery and a variety of other outdoor and indoor wood applications (Syrjanen and Oy, 2001).

The main constituents of wood are cellulose, hemicelluloses, and lignin, which are accompanied by minor amounts of extractives and inorganic compounds. The volatile organic compounds (VOCs) emitted from wood during the drying process have been well investigated (Lavery and Milota, 2000; Milota, 2000), also as a function of temperature (Banerjee et al.,1998; Su et al., 1999; Wu and Milota, 1999) and humidity (Banerjee et al., 1998; Wu and Milota, 1999). The chemical changes occurring during the actual heat-treatment were also analysed (Kotilainen, 2000). Currently, there are already some reports on VOCs emitted from heat-treated wood. Since heat-treated wood materials and products are used indoors, safety and impact of these new materials on the indoor air quality should be determined. This is especially important, as it has been found that the soluble extracts of heat-treated maritime pine and poplar timber contained potentially toxic polynuclear aromatic hydrocarbon (PAH) derivatives (Kamdem et al., 2000).

Heat treatment affects all the wood components, i.e. cellulose, hemicelluloses, lignin, and extractives. Emissions and degradation products of wood differ according to wood species. Differences can be especially detected between hardwood and softwood, which have different cell types (Sjöström, 1993).

Thermally modified wood is less hygroscopic and more dimensionally stable.
This has been attributed to degradation of hemicelluloses and to condensation reactions that hinder the uptake of moisture by forming new bonds between wood polymers such as lignin (Alén et al., 2002; Sivonen et al., 2002; Windeisen et al., 2007; Tjeerdsma and Militz, 2005). Degradation of hemicelluloses may take place via formation of soluble carbohydrates, and formation of volatile products (Alén et al., 2002; Windeisen and Wegener, 2009). Furans are well known thermal degradation products of polysaccharides; for example furfural (F) and 5-(hydroxymethyl) furfural (HMF) are abundant (Alén et al., 2002; Peters et al., 2008).

Long and Wang (2007) investigated the emissions from four common woods (spruce, poplar, masson pine, and Eucalyptus urophylla) at room temperature using high-performance liquid chromatography and gas chromatography, and the results revealed the principles of release for aldehydes and terpenes. Similarly, Hyttinen et al. (2010) compared the VOCs emitted from air-dried and heat-treated Norway spruce, Scots pine, and European poplar woods in an enclosed metal chamber and discovered the effect of different treatment conditions on volatile compounds.

Furfurals have a nutty smell and contribute a lot to the odour of heat-treated wood together with other substances such as maltol or acetic acid (Miller, 1998). Furfural is very volatile and an essential part of VOCs. Furfural can also evaporate from finished products.

The aim of the research was to find differences in the amount of Volatile Organic Compounds emitted by heat-treated samples at different temperatures $\left(180^{\circ} \mathrm{C}\right.$ and $200{ }^{\circ} \mathrm{C}$ ) and untreated wood without finished surface compared to those with finished surface with the waterborne lacquer. The investigation of VOC emissions emitted by heat-treated wood has to be focused on the influence of the finished surfaces of tested samples on the amount of VOC emissions.

It is very important to find correlation between the type of waterborne lacquer used for the surface finishing and VOC emissions and also to identify individual volatile organic compounds from the thermally modified wood without finishing.

\section{MATERIALS AND METHODS 2. MATERIJALI I METODE}

The tested woods (spruce (Picea abies L. Karst.) with the density of $0.41 \mathrm{~g} / \mathrm{cm}^{3}$ and poplar (Populus alba L.) with the density of $0.39 \mathrm{~g} / \mathrm{cm}^{3}$ ) obtained from KATRES company Ltd., Czech supplier of heat-treated wood, were investigated. The pre-dried wood samples were modified at $180{ }^{\circ} \mathrm{C}$ and $200{ }^{\circ} \mathrm{C}$ in a heat treatment process. 


\subsection{Density determination of test samples}

\subsection{Određivanje gustoće uzoraka drva}

Prior to heat treatment, samples were dried in a heating oven at $103 \pm 2{ }^{\circ} \mathrm{C}$. The oven-dry weight of the samples was determined. After the heat treatment, the oven-dry weight of the same samples was re-measured. The weight loss $\left(W_{\mathrm{L}}\right)$ of the samples, caused by heat treatment, was calculated according to the following formula,

$$
W L(\%)=100\left(W_{B H}-W_{A H}\right) / W_{B H}
$$

where $W_{\mathrm{BH}}$ is the initial oven-dry weight of the sample prior to heat treatment $(\mathrm{g})$ and $W_{\mathrm{AH}}$ is the oven-dry weight of the samples after heat treatment $(\mathrm{g})$. The equilibrium moisture content (EMC) of the test samples was determined before the tests.

The air-dried density of the samples was determined according to the following formula (ISO 3131 1975),

$$
D_{12}=M_{12} / V_{12}\left(\mathrm{~g} / \mathrm{cm}^{3}\right)
$$

where $M$ is the sample weight $(\mathrm{g})$ and $V$ is the sample volume $\left(\mathrm{cm}^{3}\right)$

Untreated and heat-treated wood samples were prepared from the same tree. Air-dried wood samples were taken from the normal manufacturing process, wrapped in aluminium foils and delivered to the test laboratory.

The samples were cut into pieces with dimensions $740 \mathrm{~mm} \times 40 \mathrm{~mm} \times 2 \mathrm{~mm}$ (longitudinal, tangential, axial) and then divided into two halves.

The first half of these samples was put into the test chamber. The samples of heat-treated material were obtained directly from the production of heattreated wood. These wood samples were also divided into two halves.

One half of these heat-treated samples were put into the chamber (ISO 16000-9, 2007) immediately after the delivery from the plant, where they were treated.

The second halves of untreated as well as heat treated samples were finished by waterborne lacquer (with non-volatile share of $35 \%$ ).

Acryl-polyurethane water dispersion is achieved with the addition of special binders and water repellents. This coating system contains about $10 \%$ of 2-buthoxyethanol (CAS: 111-76-2); $0.05 \%$ of Benzisothiazolinon (CAS: 2634-33-5); $0.1 \%$ of Methylisothiazolinon (CAS: 2682-20-4); $0.015 \%$ of reaction mixture 5-chlor-2-methylisothiazol-3(2H)- on and 2methylisothiazol-3(2H)-on (CAS:55965-84-9).

The finished samples were placed into the chamber three hours after finishing.

\subsection{Methodology of research}

2.2. Metodologija istraživanja

In step 1, the samples of the air-dried wood (spruce and poplar) with an area of $1 \mathrm{~m}^{2}$ were prepared for testing. Then, VOC emission emitted by different wood samples was collected into the desorption tubes on the sorbent Tenax TA (ISO 16000 part 1 and 5, 2007).

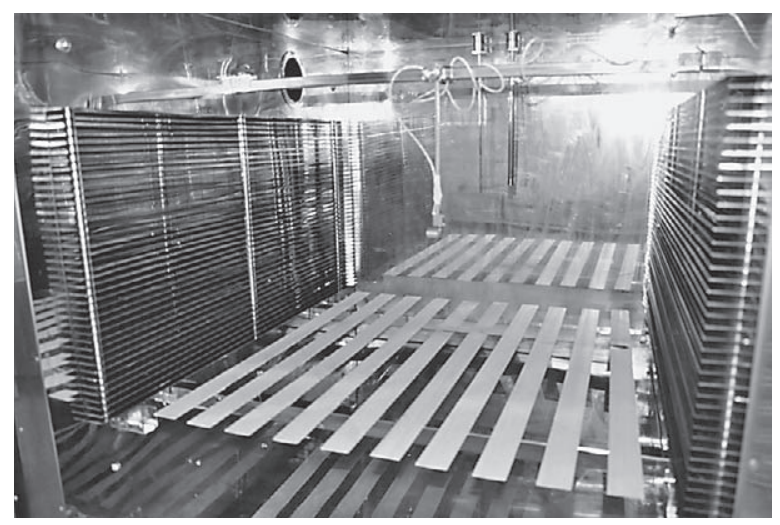

Figure 1 Testing sample in small space chamber Slika 1. Ispitni uzorci u maloj komori

By using a gas chromatograph with a mass spectrometer and a thermal desorption, the VOC emission structure was determined along with the amount of individual emitted compounds trapped in the steel sampling tube. The conducted analyses provide qualitative and quantitative data on the concentrations of selected VOC and the total volatile organic compounds (TVOC) in $\mu \mathrm{g} \cdot \mathrm{m}^{-3}$.

In step 2, the pre-dried wood samples were modified at $180^{\circ} \mathrm{C}$ and $200{ }^{\circ} \mathrm{C}$ in a heat treatment process. Then, VOC emission from heat treated wood was collected using the same methods as in the previous case.

In step 3, the samples of heat treated wood were finished by waterborne lacquer (brush application), whereby deposition of coating films was selected in the amount of $120 \mathrm{~g} \cdot \mathrm{m}^{-2}$. The curing process of this coating system is carried out using a chemical reaction (crosslinking) and evaporation of the solvent (water). The saturation time of the coating film is 2-3 hours (depending on the method of application of the coating system). Measurement of VOC emissions from test samples started 3 hours after finishing. The thickness of the dry coating film was detected by ultrasonic coating thickness gage (PosiTector 200) in range 60-70 $\mu \mathrm{m}$.

\subsection{Thermal modification process}

2.3. Proces toplinske obrade

Thermal modification was carried out using a small-scale laboratory heat treatment chamber (Katres company Ltd., Czech Republic) at 180 or $200^{\circ} \mathrm{C}$. The schedule of the five-stage thermall modification process throughout 50 hours was achieved with a temperature control system. Data of the heat-treatment process was monitored by (wet and dry bulb) thermometer, consisting of two thermometers, one dry and the other kept moist with water on a sock. Values give an indication of atmospheric humidity i.e. psychrometer diference.

The maximum temperatures $\left(180\right.$ or $\left.200{ }^{\circ} \mathrm{C}\right)$ were maintained for $3 \mathrm{~h}$.

\subsection{Air samples \\ 2.4. Uzorci zraka}

In the present study, air samples from tested samples were collected onto Tenax TA adsorbent (sampling rate $200 \mathrm{~mL} \cdot \mathrm{min}^{-1}$, time $180 \mathrm{~min}$ ) from small space 
chamber (volume of $1 \mathrm{~m}^{3}$ ). Air samples were analysed with a gas chromatograph (HP 6890) equipped with a mass selective detector (MSD 5973) after thermal desorption at $250{ }^{\circ} \mathrm{C}$ for $3 \mathrm{~min}$ (Scientific Instrument Services TD4). The column was HP-5MS (column length $30 \mathrm{~m}$, i.d. $0.25 \mathrm{~mm}$, film thickness $1 \mu \mathrm{m}$ ), and the identification of the compounds was accomplished by retention times, standard compounds, and GC-MS data library (NIS 05).

The total VOC emission was first calculated by combining the peak areas of all identified compounds, after which the relative proportion of individual compounds was calculated from the total emission. The TVOC value is defined to be the integrated detector response value in toluene equivalents of compounds eluting between and including $\mathrm{C}_{6}$ to $\mathrm{C}_{16}$ as given in ISO 16000-6.
VOC emissions from test samples were measured as a function of time (time from insertion of test sample into the test chamber).

\section{RESULTS}

\section{REZULTATI}

This research deals with VOC emissions emitted by solid Spruce (Picea abies) and Poplar (Populus alba) wood treated at $200{ }^{\circ} \mathrm{C}$ and $180{ }^{\circ} \mathrm{C}$. The emissions of Volatile Organic Compounds from heat treated wood and air-dried (natural) wood were compared with GC-MS analysis.

VOC emissions from test samples were measured as a function of time (time from insertion of test sample into the test chamber). VOC emissions from natural wood, heat treated wood and finished surface of

Table 1 VOCs emitted by tested sample of untreated wood - spruce

Tablica 1. Emisija VOC-a iz uzoraka netretirane smrekovine

\begin{tabular}{|c|c|c|c|c|c|}
\hline & $\begin{array}{l}\text { Compounds } \\
\text { Kemijski spoj }\end{array}$ & $\begin{array}{l}\text { After } 3 \text { hours }{ }^{\mathrm{a}} \\
\text { Nakon } 3 \text { sata }\end{array}$ & $\begin{array}{l}\text { After } 24 \text { hours }^{\mathrm{a}} \\
\text { Nakon } 24 \text { sata }\end{array}$ & $\begin{array}{l}\text { After } 72 \text { hours }^{\mathrm{a}} \\
\text { Nakon } 72 \text { sata }\end{array}$ & $\begin{array}{l}\text { After } 672 \text { hours }^{\mathrm{a}} \\
\text { Nakon } 672 \text { sata }\end{array}$ \\
\hline & $\begin{array}{l}\text { Natural wood - untreated } \\
\text { Prirodno, netretirano drvo }\end{array}$ & \multicolumn{4}{|c|}{ Spruce wood / Drvo smreke } \\
\hline No. & Unit / Jedinica & $\mu \mathrm{g} / \mathrm{m}^{3}$ & $\mu \mathrm{g} / \mathrm{m}^{3}$ & $\mu \mathrm{g} / \mathrm{m}^{3}$ & $\mu \mathrm{g} / \mathrm{m}^{3}$ \\
\hline 1 & Ethyl Acetate & $<0.1$ & $<0.1$ & $(0.2 \pm 0.1)$ & $<0.1$ \\
\hline 2 & Benzene & $<0.1$ & $(0.2 \pm 0.1)$ & $(0.2 \pm 0.1)$ & $(0.2 \pm 0.1)$ \\
\hline 3 & 1-Methoxy-2-Propanol & $(0.3 \pm 0.1)^{b}$ & $(0.1 \pm 0.03)$ & $<0.1$ & $(0.8 \pm 0.2)$ \\
\hline 4 & Pentanal & $(2 \pm 0.6)$ & $(1.3 \pm 0.4)$ & $(1.1 \pm 0.3)$ & $(0.6 \pm 0.2)$ \\
\hline 5 & Trichlorethylene & $<0.1$ & $<0.1$ & $<0.1$ & $<0.1$ \\
\hline 6 & Toluene & $(1 \pm 0.3)$ & $(1 \pm 0.3)$ & $(1.2 \pm 0.4)$ & $(2 \pm 0.6)$ \\
\hline 7 & Hexanal & $(5.4 \pm 1.6)$ & $(4.4 \pm 1.3)$ & $(3.8 \pm 1.1)$ & $(2.1 \pm 0.6)$ \\
\hline 8 & Tetrachloretylene & $<0.1$ & $(0.2 \pm 0.1)$ & $(0.2 \pm 0.1)$ & $(0.2 \pm 0.1)$ \\
\hline 9 & n-Butyl acetate & $(1.3 \pm 0.4)$ & $(0.4 \pm 0.1)$ & $(0.9 \pm 0.3)$ & $(0.9 \pm 0.3)$ \\
\hline 10 & Furfural & $(7.8 \pm 2.3)$ & $(2.5 \pm 0.8)$ & $(1.8 \pm 0.5)$ & $(1 \pm 0.3)$ \\
\hline 11 & Ethylbenzene & $(0.7 \pm 0.2)$ & $(0.2 \pm 0.1)$ & $(0.5 \pm 0.2)$ & $(0.7 \pm 0.2)$ \\
\hline 12 & $\mathrm{~m}, \mathrm{p}$-Xylene & $(1.8 \pm 0.5)$ & $(0.8 \pm 0.2)$ & $(1.8 \pm 0.5)$ & $(2 \pm 0.6)$ \\
\hline 13 & Styrene & $<0.1$ & $(0.1 \pm 0.03)$ & $<0.1$ & $<0.1$ \\
\hline 14 & o-Xylene & $(0.4 \pm 0.1)$ & $(0.1 \pm 0.03)$ & $(0.4 \pm 0.1)$ & $(0.4 \pm 0.1)$ \\
\hline 15 & Butoxy-Ethanol & $(0.3 \pm 0.1)$ & $(0.1 \pm 0.03)$ & $<0.1$ & $<0.1$ \\
\hline 16 & $\alpha$-Pinene & $(0.1 \pm 0.03)$ & $(0.1 \pm 0.03)$ & $(0.1 \pm 0.03)$ & $(0.3 \pm 0.1)$ \\
\hline 17 & Camphene & $<0.1$ & $<0.1$ & $<0.1$ & $(0.2 \pm 0.1)$ \\
\hline 18 & 3-Ethyl-Toluene & $(0.3 \pm 0.1)$ & $(0.3 \pm 0.1)$ & $(0.3 \pm 0.1)$ & $(0.3 \pm 0.1)$ \\
\hline 19 & 4-Ethyl-Toluene & $(0.3 \pm 0.1)$ & $(0.3 \pm 0.1)$ & $(0.4 \pm 0.1)$ & $(0.3 \pm 0.1)$ \\
\hline 20 & 1,3,5-Trimethyl-Benzene & $<0.1$ & $<0.1$ & $<0.1$ & $<0.1$ \\
\hline 21 & Phenol & $(0.7 \pm 0.2)$ & $(0.5 \pm 0.2)$ & $(0.5 \pm 0.2)$ & $(0.3 \pm 0.1)$ \\
\hline 22 & $\beta$-Pinene & $(0.4 \pm 0.1)$ & $(0.4 \pm 0.1)$ & $(0.2 \pm 0.1)$ & $(0.2 \pm 0.1)$ \\
\hline 23 & 2-Ethyl Toluene & $(0.1 \pm 0.03)$ & $(0.1 \pm 0.03)$ & $(0.1 \pm 0.03)$ & $(0.1 \pm 0.03)$ \\
\hline 24 & Myrcene & $<0.1$ & $<0.1$ & $<0.1$ & $<0.1$ \\
\hline 25 & 1,2,4-Trimethyl-Benzene & $<0.1$ & $(0.2 \pm 0.1)$ & $(0.2 \pm 0.1)$ & $(0.2 \pm 0.1)$ \\
\hline 26 & $\alpha$-Phellandrene & $<0.1$ & $<0.1$ & $<0.1$ & $<0.1$ \\
\hline 27 & 3- $\delta$-Carene & $(0.6 \pm 0.2)$ & $(0.4 \pm 0.1)$ & $(0.4 \pm 0.1)$ & $(0.4 \pm 0.1)$ \\
\hline 28 & 1,2,3-Trimethyl-Benzene & $(0.1 \pm 0.03)$ & $(0.1 \pm 0.03)$ & $(0.1 \pm 0.03)$ & $(0.1 \pm 0.03)$ \\
\hline 29 & Limonene & $(1.1 \pm 0.3)$ & $(0.4 \pm 0.1)$ & $(0.4 \pm 0.1)$ & $(0.1 \pm 0.03)$ \\
\hline 30 & $\gamma$-Terpinene & $<0.1$ & $<0.1$ & $<0.1$ & $<0.1$ \\
\hline 31 & Bornyl Acetate & $<0.1$ & $<0.1$ & $<0.1$ & $<0.1$ \\
\hline 32 & TVOC $_{\mathrm{MS}}{ }^{\mathrm{c}}$ & $(44 \pm 13)$ & $(22 \pm 7)$ & $(19 \pm 6)$ & $(17 \pm 5)$ \\
\hline
\end{tabular}

a Time of measurement (time from insertion of test sample into the test chamber) / vrijeme mjerenja (vrijeme od stavljanja uzoraka $u$ komoru do vremena provedbe mjerenja); ${ }^{b}$ results are shown as average result ${ }^{ \pm}$expanded measurement uncertainty / rezultati su prikazani kao prosječna vrijednost rezultata \pm proširena mjerna nesigurnost; ${ }^{\mathrm{c}} \mathrm{TVOC}_{\mathrm{MS}}$ - Total Volatile Organic Compounds determined by Mass Spectrometry / ukupna emisija hlapljivih organskih spojeva određena masenom spektrometrijom 
Table 2 VOCs emitted by tested sample of untreated wood - poplar

Tablica 2. Emisija VOC-a iz uzoraka netretirane topolovine

\begin{tabular}{|c|c|c|c|c|c|}
\hline & $\begin{array}{l}\text { Compounds } \\
\text { Kemijski spoj }\end{array}$ & $\begin{array}{l}\text { After } 3 \text { hours }{ }^{\mathrm{a}} \\
\text { Nakon } 3 \text { sata }\end{array}$ & $\begin{array}{l}\text { After } 24 \text { hours }^{\text {a }} \\
\text { Nakon } 24 \text { sata }\end{array}$ & $\begin{array}{l}\text { After } 72 \text { hours } \\
\text { Nakon } 72 \text { sata }\end{array}$ & $\begin{array}{c}\text { After } 672 \text { hours }^{\mathrm{a}} \\
\text { Nakon } 672 \text { sata }\end{array}$ \\
\hline & $\begin{array}{l}\text { Natural wood - untreated } \\
\text { Prirodno, netretirano drvo }\end{array}$ & \multicolumn{4}{|c|}{$\begin{array}{l}\text { Poplar wood } \\
\text { Drvo topole }\end{array}$} \\
\hline No. & Unit / Jedinica & $\mu \mathrm{g} / \mathrm{m}^{3}$ & $\mu \mathrm{g} / \mathrm{m}^{3}$ & $\mu \mathrm{g} / \mathrm{m}^{3}$ & $\mu \mathrm{g} / \mathrm{m}^{3}$ \\
\hline 1 & Ethyl Acetate & $(0.2 \pm 0.1)^{\mathrm{b}}$ & $(0.4 \pm 0.1)$ & $<0.1$ & $<0.1$ \\
\hline 2 & Benzene & $<0.1$ & $<0.1$ & $<0.1$ & $<0.1$ \\
\hline 3 & 1-Methoxy-2-Propanol & $(0.1 \pm 0.03)$ & $<0.1$ & $<0.1$ & $(0.8 \pm 0.2)$ \\
\hline 4 & Pentanal & $(1.6 \pm 0.5)$ & $(0.8 \pm 0.2)$ & $(0.3 \pm 0.1)$ & $(0.3 \pm 0.1)$ \\
\hline 5 & Trichlorethylene & $<0.1$ & $<0.1$ & $<0.1$ & $<0.1$ \\
\hline 6 & Toluene & $(0.8 \pm 0.2)$ & $(0.7 \pm 0.2)$ & $(0.5 \pm 0.2)$ & $(0.7 \pm 0.2)$ \\
\hline 7 & Hexanal & $(10.1 \pm 3)$ & $(5.1 \pm 1.5)$ & $(0.9 \pm 0.3)$ & $(0.8 \pm 0.2)$ \\
\hline 8 & Tetrachloretylene & $<0.1$ & $<0.1$ & $(0.2 \pm 0.1)$ & $(0.2 \pm 0.1)$ \\
\hline 9 & n-Butyl acetate & $(1.4 \pm 0.4)$ & $(0.4 \pm 0.1)$ & $(0.8 \pm 0.2)$ & $(0.8 \pm 0.2)$ \\
\hline 10 & Furfural & $(1.3 \pm 0.4)$ & $(1 \pm 0.3)$ & $(0.3 \pm 0.1)$ & $(0.3 \pm 0.1)$ \\
\hline 11 & Ethylbenzene & $(0.7 \pm 0.2)$ & $(0.2 \pm 0.1)$ & $(0.2 \pm 0.1)$ & $(0.4 \pm 0.1)$ \\
\hline 12 & m,p-Xylene & $(2.2 \pm 0.7)$ & $(0.7 \pm 0.2)$ & $(0.8 \pm 0.2)$ & $(1.2 \pm 0.4)$ \\
\hline 13 & Styrene & $<0.1$ & $(0.1 \pm 0.03)$ & $<0.1$ & $(0.1 \pm 0.03)$ \\
\hline 14 & o-Xylene & $(0.4 \pm 0.1)$ & $(0.1 \pm 0.03)$ & $(0.1 \pm 0.03)$ & $(0.3 \pm 0.1)$ \\
\hline 15 & Butoxy-Ethanol & $(0.1 \pm 0.03)$ & $<0.1$ & $<0.1$ & $<0.1$ \\
\hline 16 & $\alpha$-Pinene & $<0.1$ & $<0.1$ & $(0.1 \pm 0.03)$ & $(0.1 \pm 0.03)$ \\
\hline 17 & Camphene & $<0.1$ & $<0.1$ & $<0.1$ & $<0.1$ \\
\hline 18 & 3-Ethyl-Toluene & $(0.1 \pm 0.03)$ & $(0.1 \pm 0.03)$ & $<0.1$ & $(0.1 \pm 0.03)$ \\
\hline 19 & 4-Ethyl-Toluene & $(0.3 \pm 0.1)$ & $(0.3 \pm 0.1)$ & $(0.1 \pm 0.03)$ & $(0.1 \pm 0.03)$ \\
\hline 20 & 1,3,5-Trimethyl-Benzene & $<0.1$ & $<0.1$ & $<0.1$ & $<0.1$ \\
\hline 21 & Phenol & $(0.7 \pm 0.2)$ & $(0.8 \pm 0.2)$ & $(0.3 \pm 0.1)$ & $(0.5 \pm 0.2)$ \\
\hline 22 & $\beta$-Pinene & $(0.1 \pm 0.03)$ & $(0.1 \pm 0.03)$ & $(0.1 \pm 0.03)$ & $(0.1 \pm 0.03)$ \\
\hline 23 & 2-Ethyl Toluene & $(0.1 \pm 0.03)$ & $(0.1 \pm 0.03)$ & $(0.1 \pm 0.03)$ & $(0.1 \pm 0.03)$ \\
\hline 24 & Myrcene & $<0.1$ & $<0.1$ & $<0.1$ & $<0.1$ \\
\hline 25 & 1,2,4-Trimethyl-Benzene & $<0.1$ & $<0.1$ & $<0.1$ & $<0.1$ \\
\hline 26 & $\alpha$-Phellandrene & $<0.1$ & $<0.1$ & $<0.1$ & $<0.1$ \\
\hline 27 & 3- $\delta$-Carene & $(0.1 \pm 0.03)$ & $(0.1 \pm 0.03)$ & $(0.1 \pm 0.03)$ & $(0.2 \pm 0.1)$ \\
\hline 28 & 1,2,3-Trimethyl-Benzene & $(0.1 \pm 0.03)$ & $(0.1 \pm 0.03)$ & $(0.1 \pm 0.03)$ & $(0.1 \pm 0.03)$ \\
\hline 29 & Limonene & $(0.1 \pm 0.03)$ & $(0.1 \pm 0.03)$ & $(0.1 \pm 0.03)$ & $(0.1 \pm 0.03)$ \\
\hline 30 & $\gamma$-Terpinene & $<0.1$ & $<0.1$ & $<0.1$ & $<0.1$ \\
\hline 31 & Bornyl Acetate & $<0.1$ & $<0.1$ & $<0.1$ & $<0.1$ \\
\hline 32 & TVOC $_{\mathrm{MS}}{ }^{\mathrm{c}}$ & $(24 \pm 7)$ & $(13 \pm 4)$ & $(10 \pm 3)$ & $(8 \pm 2)$ \\
\hline
\end{tabular}

${ }^{a}$ Time of measurement (time from insertion of test sample into the test chamber) / vrijeme mjerenja (vrijeme od stavljanja uzoraka u komoru do vremena provedbe mjerenja); ${ }^{\mathrm{b}}$ results are shown as average result ${ }^{ \pm}$expanded measurement uncertainty / rezultati su prikazani kao prosječna

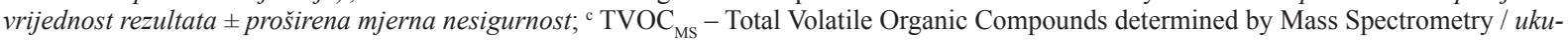
pna emisija hlapljivih organskih spojeva određena masenom spektrometrijom

heat treated wood were monitored during one month (i.e. 28 days) according the standard CSN EN ISO $16000-9$.

The results of measurements of emissions emitted by native spruce and poplar in dependence of time after the sample preparation are shown in Tables 1 and 2.

The surface of natural wood samples was treated by grinding before insertion of the test sample into the test chamber. Therefore, the wood structure was disturbed. This fact caused gradual release of chemicals, especially aldehydes (Pentanal, Hexanal). The values of TVOC is decreasing in time.

Based on the obtained results (Tables 1 to 4), it is concluded that heat-treatment of wood increases the quantity of VOC emissions emitted by tested samples. The main difference was found in the amount of emitted furfural and phenol in the gaseous blend evaporated by heat-treated spruce and poplar in normal conditions.
The temperature of heat-treatment has a great influence on the treated wood.

The higher the temperature during spruce heattreatment, the higher are the furfural emissions. Furfural and phenol are typical chemicals produced as a result of thermal degradation of wood components.

The surface finished by waterborne lacquer does not decrease the amount of emissions escaping from heat-treated spruce and poplar wood. Surprisingly, waterborne lacquers even elevated the amount of VOCs.

Figures 2 and 4 show the influence of wood modification temperature and of the time between the VOC measurement and wood modification. The amount of VOC emission decreases with the decreasing temperature of wood modification. The amount of emitted VOC declines with the increasing time between wood modification and measurement of VOC emissions emitted by tested samples. Heat-treated spruce wood 


\begin{tabular}{|c|c|c|c|c|c|c|c|c|c|c|c|c|c|c|c|c|c|c|c|c|c|c|c|}
\hline $\begin{array}{l}\sqrt{N} \\
\hat{\sigma} \\
\vdots \\
0 \\
z \\
z \\
z\end{array}$ & 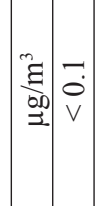 & $\begin{array}{l}\overrightarrow{0} \\
\mathrm{v}\end{array}$ & 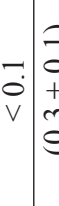 & 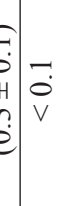 & $\begin{array}{l}\widehat{\jmath} \\
0 \\
+1 \\
\infty \\
\vdots \\
e\end{array}$ & 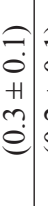 & 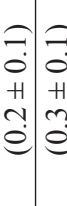 & 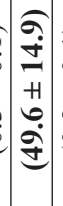 & 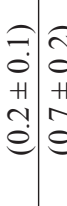 & 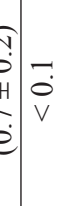 & 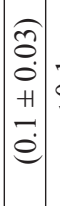 & & $\begin{array}{l}\overrightarrow{0} \\
\mathrm{O}\end{array}$ & 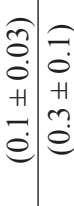 & 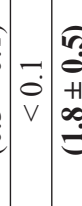 & 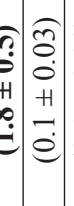 & $\mid$\begin{tabular}{c}
$\sigma$ \\
$\delta$ \\
0 \\
+ \\
\hdashline \\
$\dot{e}$
\end{tabular} & \begin{tabular}{l|l} 
& \\
$\dot{0}$ & $\overrightarrow{0}$ \\
$v$ & $V$
\end{tabular} & 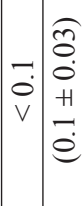 & 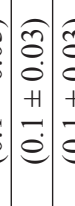 & & & $\begin{array}{l}\infty \\
+1 \\
\end{array}$ \\
\hline 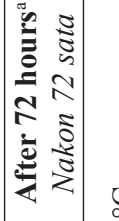 & 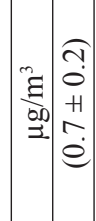 & 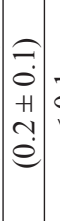 & 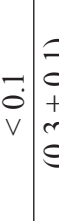 & & $\begin{array}{l}\widehat{a} \\
0 \\
+ \\
0 \\
\infty \\
\dot{e}\end{array}$ & $\begin{array}{l}0 \\
0 \\
0 \\
+ \\
m \\
\vdots \\
e\end{array}$ & 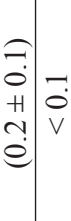 & 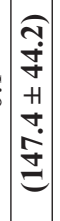 & 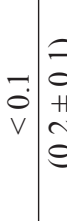 & 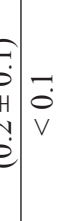 & 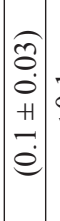 & 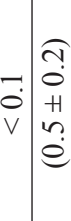 & 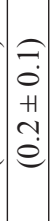 & 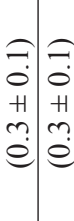 & 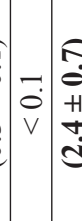 & 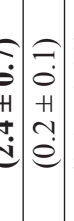 & $\begin{array}{c}\hat{\sigma} \\
0 \\
0 \\
+1 \\
\tilde{e}\end{array}$ & 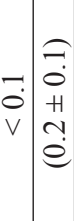 & 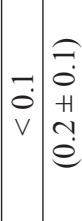 & {$\left[\begin{array}{c}\widehat{\sigma} \\
0 \\
0 \\
+ \\
-\dot{e}\end{array}\right.$} & $\begin{array}{l}\tilde{0} \\
0 \\
0 \\
+1 \\
-\dot{0} \\
\end{array}$ & & $\begin{array}{l}6 \\
\stackrel{6}{1} \\
+1 \\
6\end{array}$ \\
\hline 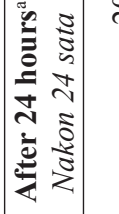 & 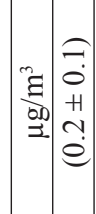 & - & 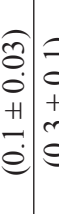 & 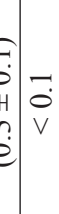 & $\begin{array}{c}- \\
\overrightarrow{0} \\
+ \\
0 \\
e \\
e\end{array}$ & $\begin{array}{l}\hat{\sigma} \\
\text { o. } \\
+1 \\
+1\end{array}$ & & 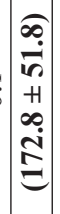 & 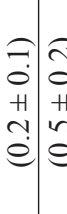 & 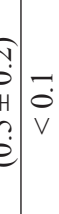 & $\begin{array}{c}- \\
\overrightarrow{0} \\
+1 \\
\tilde{e} \\
e\end{array}$ & & $\begin{array}{l}\widehat{C} \\
\dot{0} \\
+ \\
m \\
e \\
e\end{array}$ & 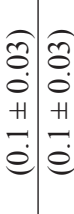 & \begin{tabular}{ll}
$Z$ \\
\hdashline \\
0 \\
0
\end{tabular} & 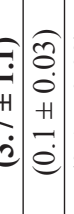 & $\mid \begin{array}{c}\hat{\sigma} \\
0 \\
0 \\
+ \\
0 \\
0\end{array}$ & 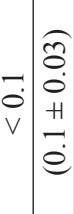 & & $\begin{array}{l}\tilde{0} \\
\dot{0} \\
+ \\
+1 \\
-\dot{e}\end{array}$ & $\begin{array}{l}0 \\
0 \\
0 \\
+1 \\
\dot{0} \\
\dot{e}\end{array}$ & & $\begin{array}{l}\underset{N}{N} \\
+1 \\
\infty\end{array}$ \\
\hline$s$ & 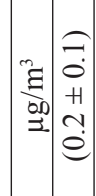 & $\begin{array}{ll}0 & \\
+ \\
N \\
\end{array}$ & 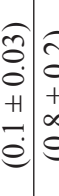 & $\begin{array}{ll} \\
\dot{b} \\
H\end{array}$ & $\begin{array}{l}\widehat{\imath} \\
\vdots \\
+1 \\
\infty \\
\dot{0} \\
\end{array}$ & & 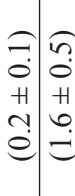 & 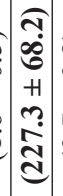 & 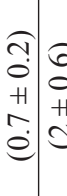 & 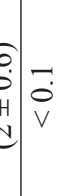 & $\begin{array}{l}0 \\
0 \\
+ \\
+ \\
\dot{e} \\
e\end{array}$ & 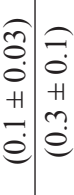 & \begin{tabular}{l}
$c$ \\
\hdashline \\
0 \\
+1 \\
$v$ \\
$e$ \\
$e$
\end{tabular} & 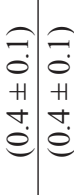 & 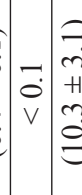 & 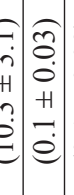 & $\begin{array}{c}\hat{\sigma} \\
0 \\
0 \\
+1 \\
\tilde{e}\end{array}$ & 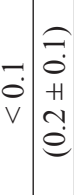 & 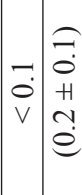 & 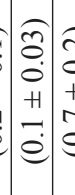 & 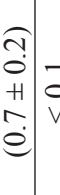 & $\begin{array}{l}- \\
\dot{0} \\
v\end{array}$ & $\underset{N}{\mathbb{N}}$ \\
\hline
\end{tabular}

告

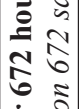

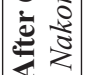

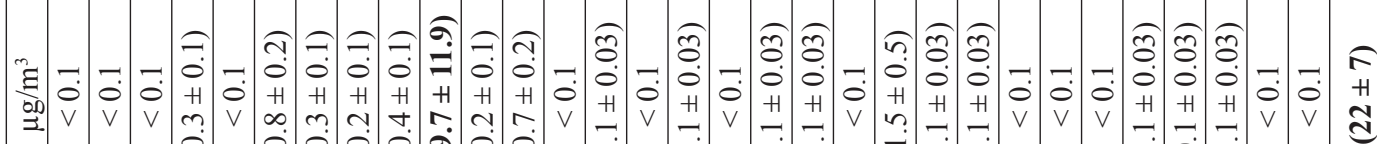

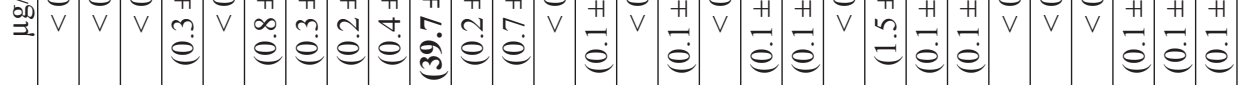

: 8

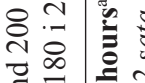

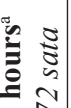

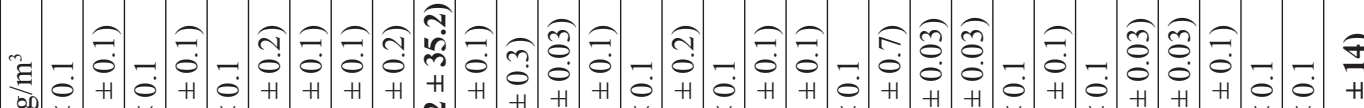

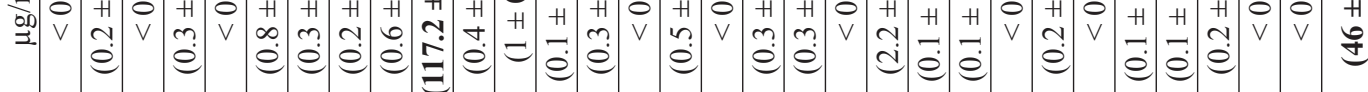

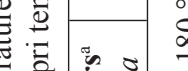

0
$\infty$

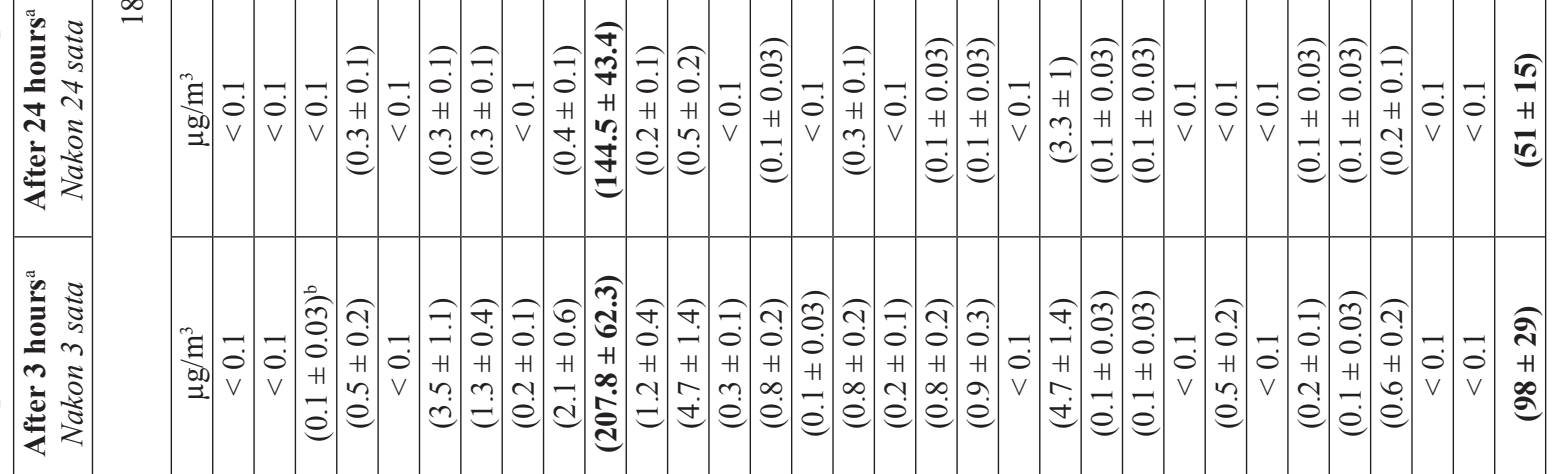

范

要

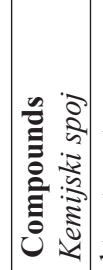

常 है

苟

完茪

券涭

药

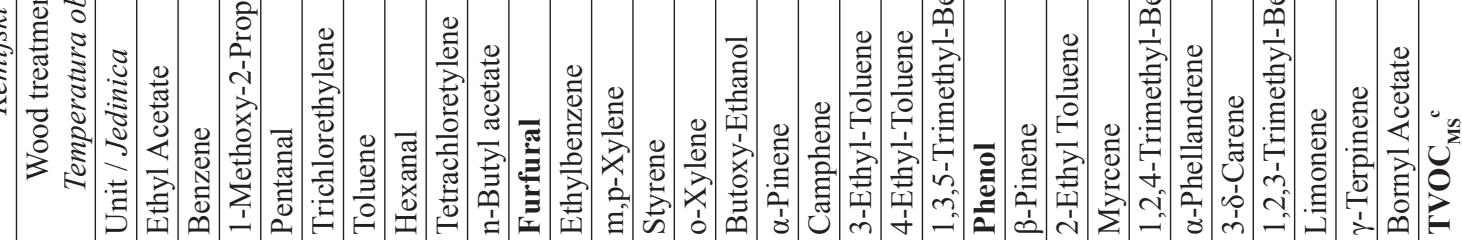

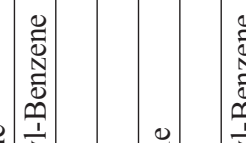

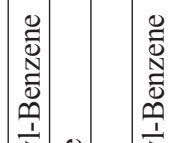

ऽ国

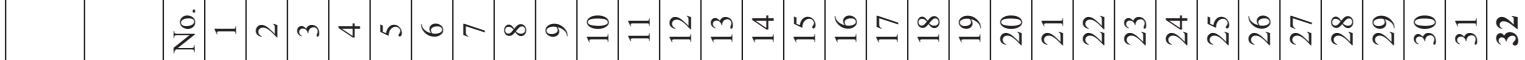




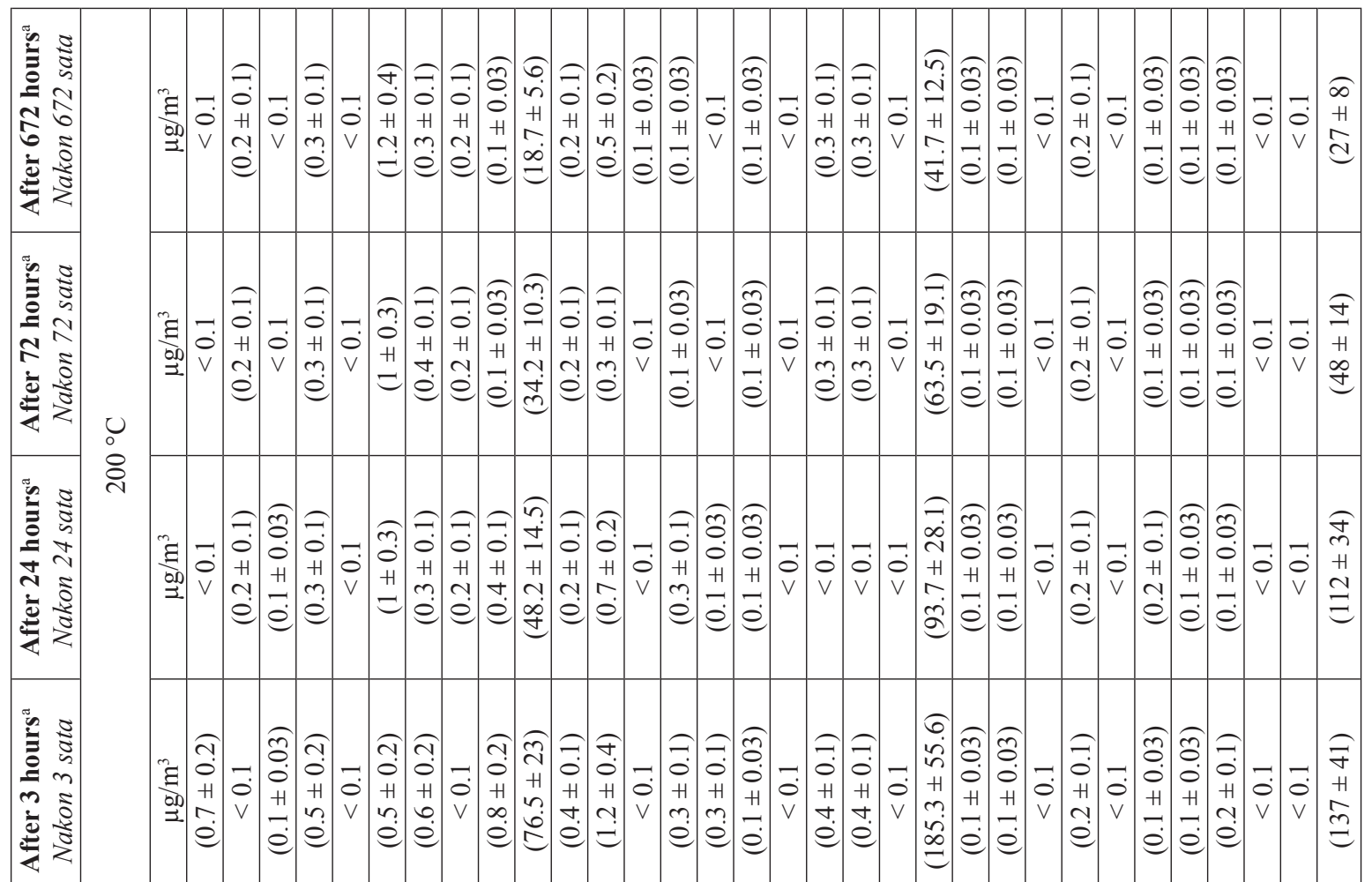

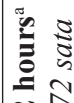

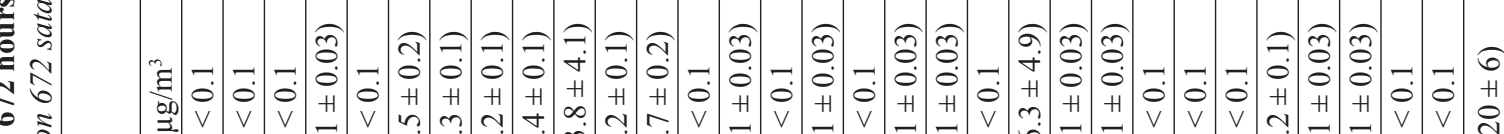

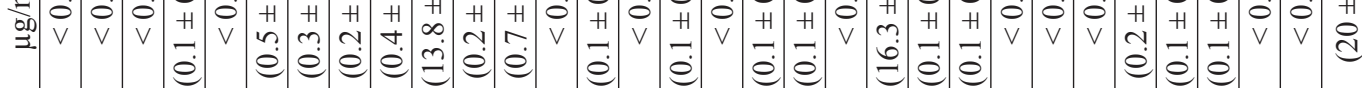

कृष

o

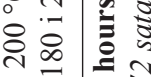

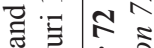

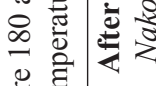

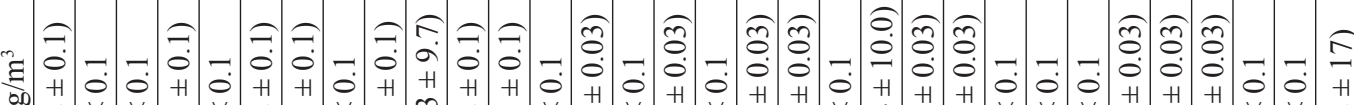

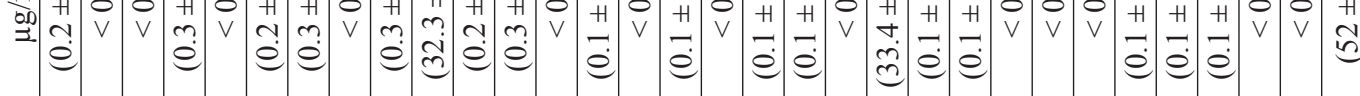

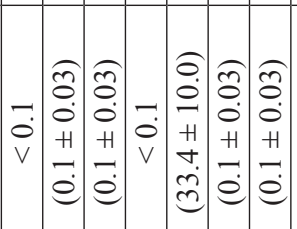

要行

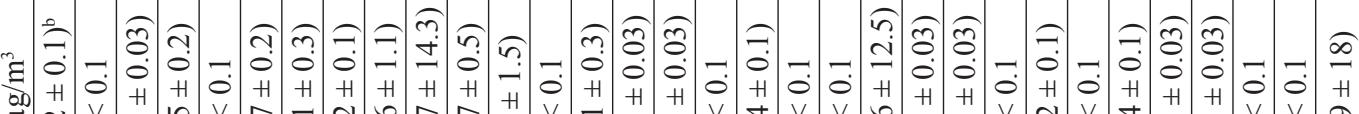

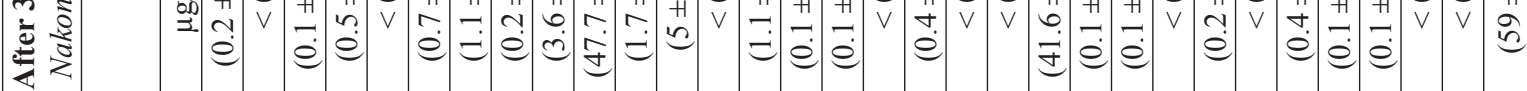




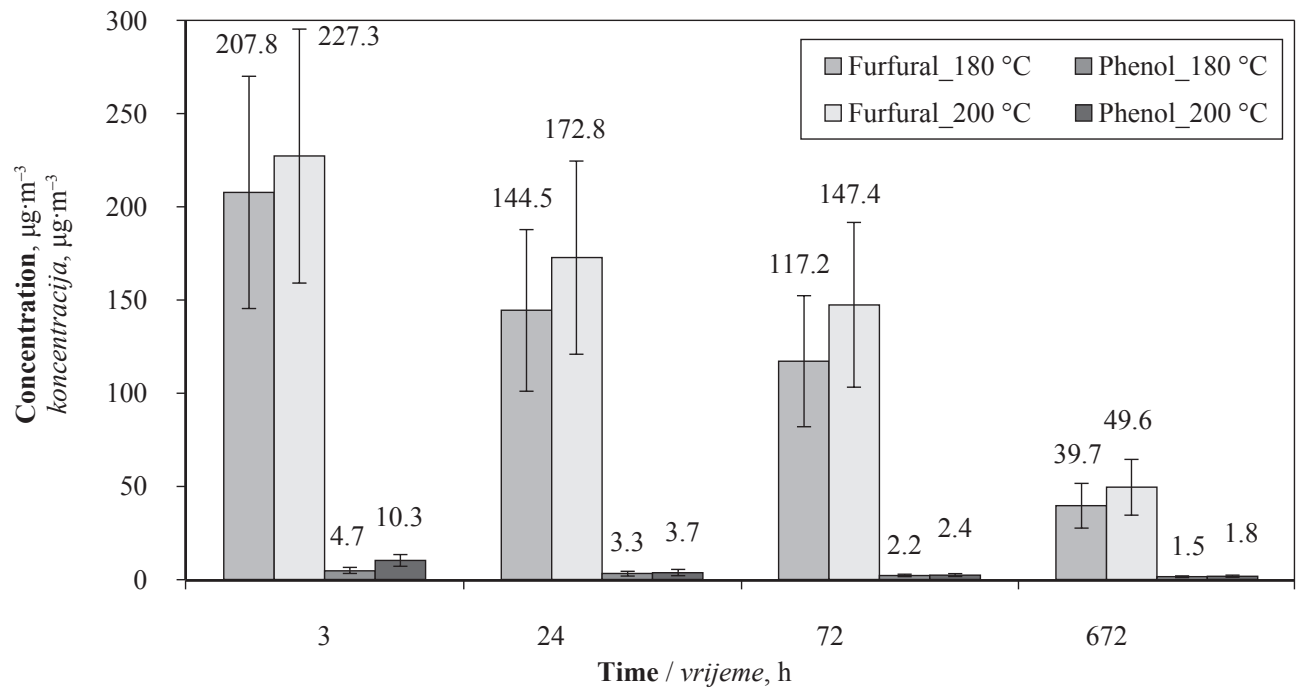

Figure 2 Amount of Furfural and Phenol emitted by heat-treated spruce wood after 3, 24, 72 and $672 \mathrm{~h}$ Slika 2. Količina furfurala i fenola koju emitira toplinski obrađena smrekovina nakon 3, 24, 72 i 672 sata

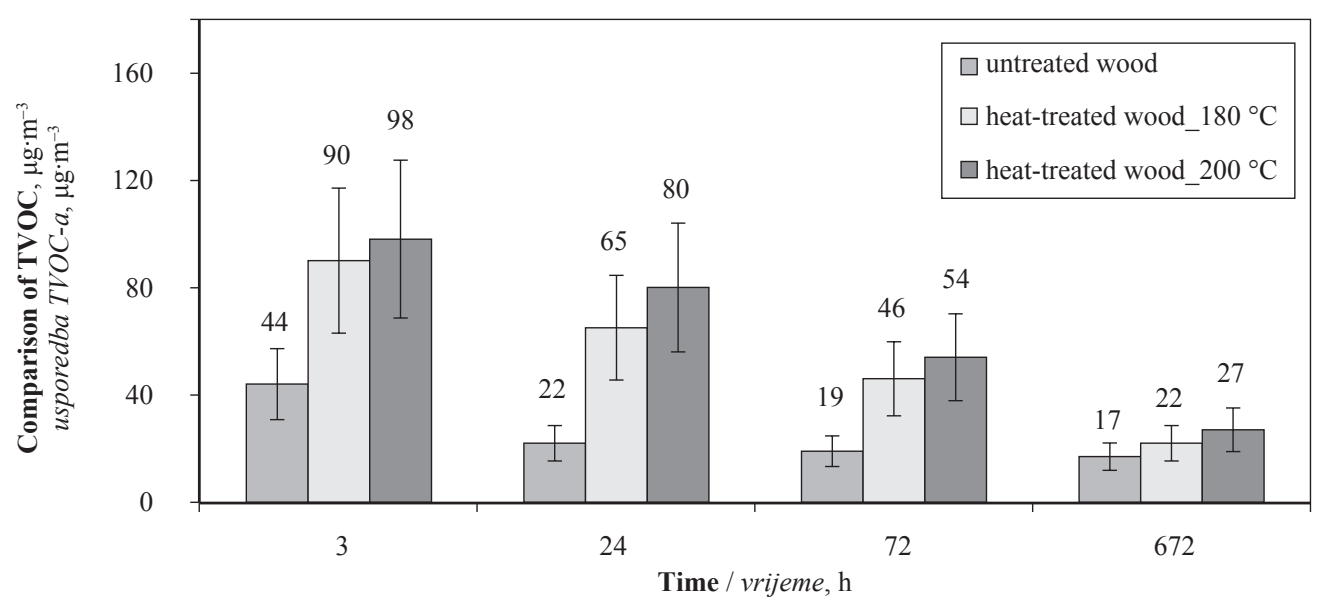

Figure 3 Comparison of TVOC from Norway Spruce - heat treated wood at $180{ }^{\circ} \mathrm{C}$ and $200{ }^{\circ} \mathrm{C}$ and untreated wood Slika 3. Usporedba TVOC-a koju emitira smrekovina - toplinski obrađena pri 180 i $200{ }^{\circ} \mathrm{C}$ i neobrađeno (prirodno) drvo

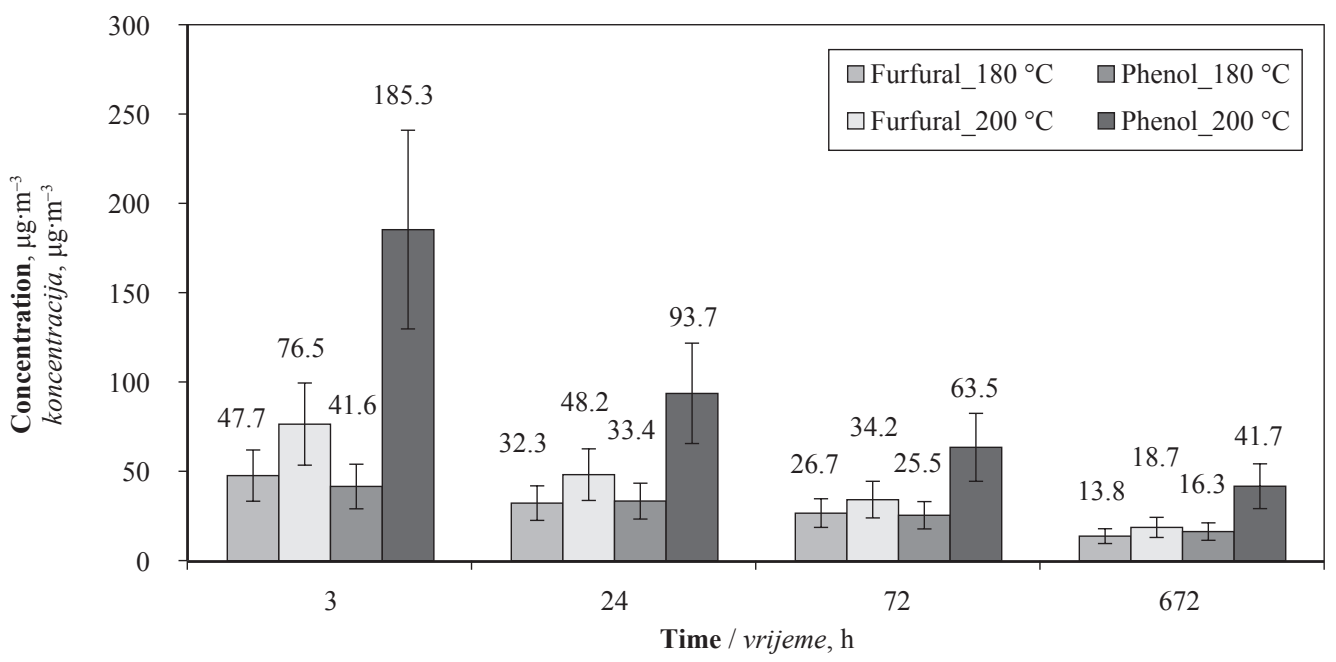

Figure 4 Amount of Furfural and Phenol emitted by heat-treated poplar wood after 3, 24, 72 and $672 \mathrm{~h}$ Slika 4. Količina furfurala i fenola koju emitira toplinski obrađena topolovina nakon 3, 24, 72 i 672 sata 


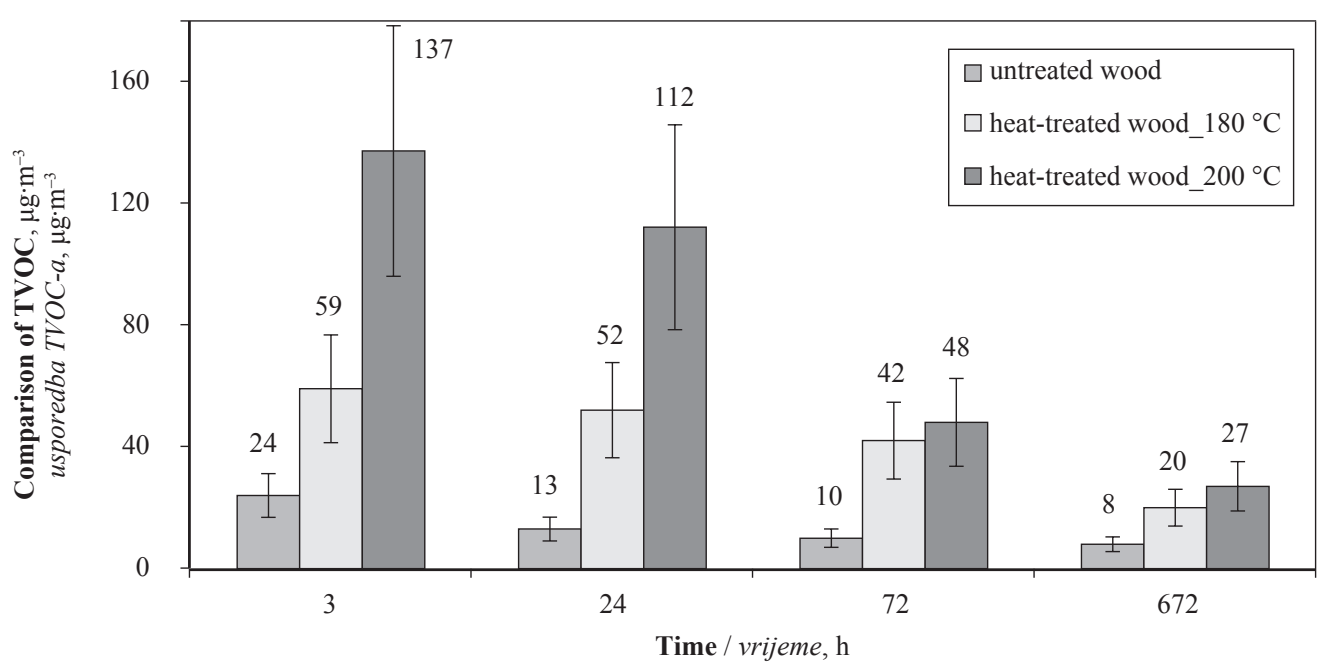

Figure 5 Comparison of TVOC from White Poplar- heat treated wood at $180{ }^{\circ} \mathrm{C}$ and $200{ }^{\circ} \mathrm{C}$ and untreated wood Slika 5. Usporedba TVOC-a koji emitira topolovina - toplinski obrađena pri 180 i $200{ }^{\circ} \mathrm{C}$ i neobrađeno (prirodno) drvo

emitted more Fufural than Phenol, while the opposite was observed with heat-treated poplar wood.

Figures 3 and 5 show a comparison of TVOC from heat treated wood - spruce (Picea abies) and poplar (Populus alba L.) at $180{ }^{\circ} \mathrm{C}$ and $200{ }^{\circ} \mathrm{C}$ and untreated wood in dependence on time. The highest concentration of TVOC was emitted by thermowood at $200{ }^{\circ} \mathrm{C}$. The measured values of TVOC decrease in time.

Figures 6 and 8 show the influence of wood modification temperature and of time between VOC measurement and surface finishing. The amount of VOC emission decreases with the decreasing temperature of wood modification. Heat treated spruce wood emitted a higher amount of furfural 3 hours after finishing $\left(299 \mu \mathrm{g} \cdot \mathrm{m}^{-3}\right)$, while heat treated poplar wood emitted higher concentration of phenol $\left(129 \mu \mathrm{g} \cdot \mathrm{m}^{-3}\right)$ after finishing.

Figures 7 and 9 present data of TVOC emitted by heat treated spruce and poplar wood before and after finishing. TVOC emitted by heat treated spruce and poplar wood significantly increased after finishing. This phenomenon can be explained by the increase of Buthoxy-ethanol, the amount of which is shown in Figures 10 and 11.

Figures 10 and 11 show the influence of wood modification temperature and of time between VOC measurement and surface finishing. The amount of Buthoxy-ethanol can be measured 3 hours after finishing. This phenomenon can be explained by the use of waterborne lacquer for finishing of heat treated wood. Waterborne lacquer used for finishing of heat treated wood contains about ten percent of this chemical (product safety data sheet).

\section{DISCUSSION \\ 4. RASPRAVA}

Based on the results of this paper, VOC emissions from natural and heat treated wood can be assessed.

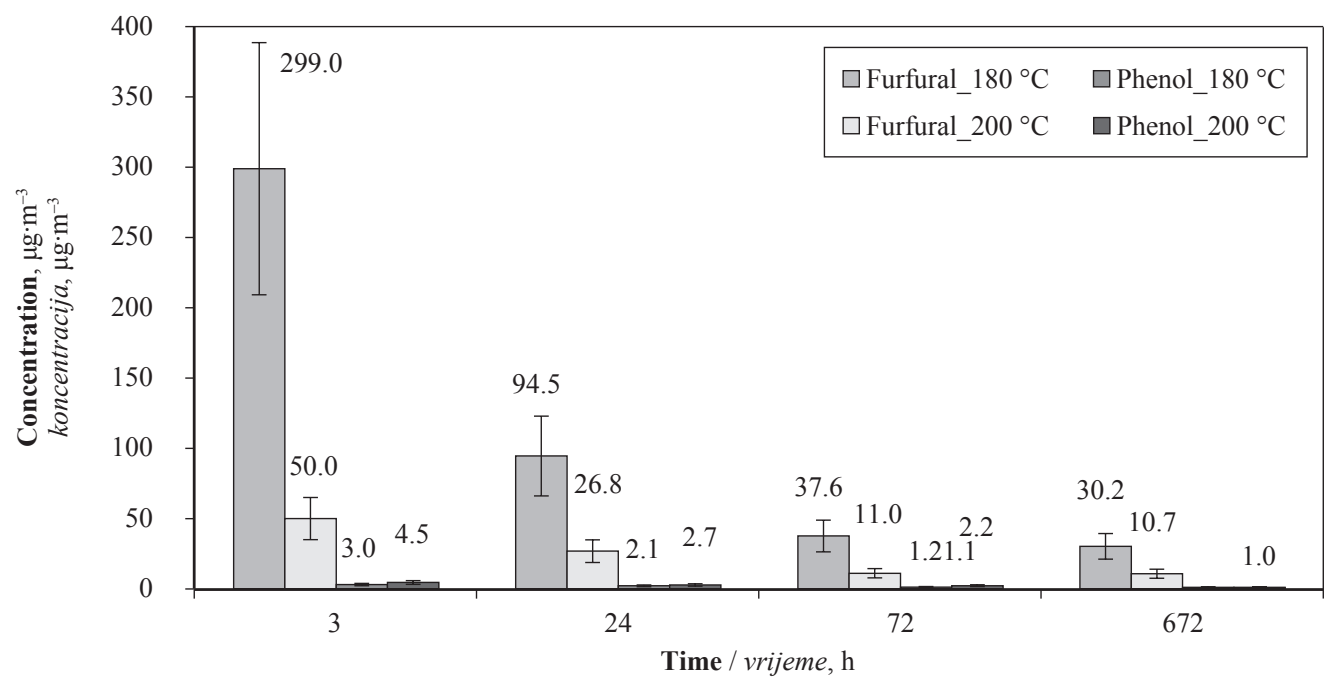

Figure 6 Amount of Furfural and Phenol emitted by heat-treated Spruce wood after finishing

Slika 6. Količina furfurala i fenola koju emitira toplinski obrađena smrekovina nakon završne obrade vodenim lakom 


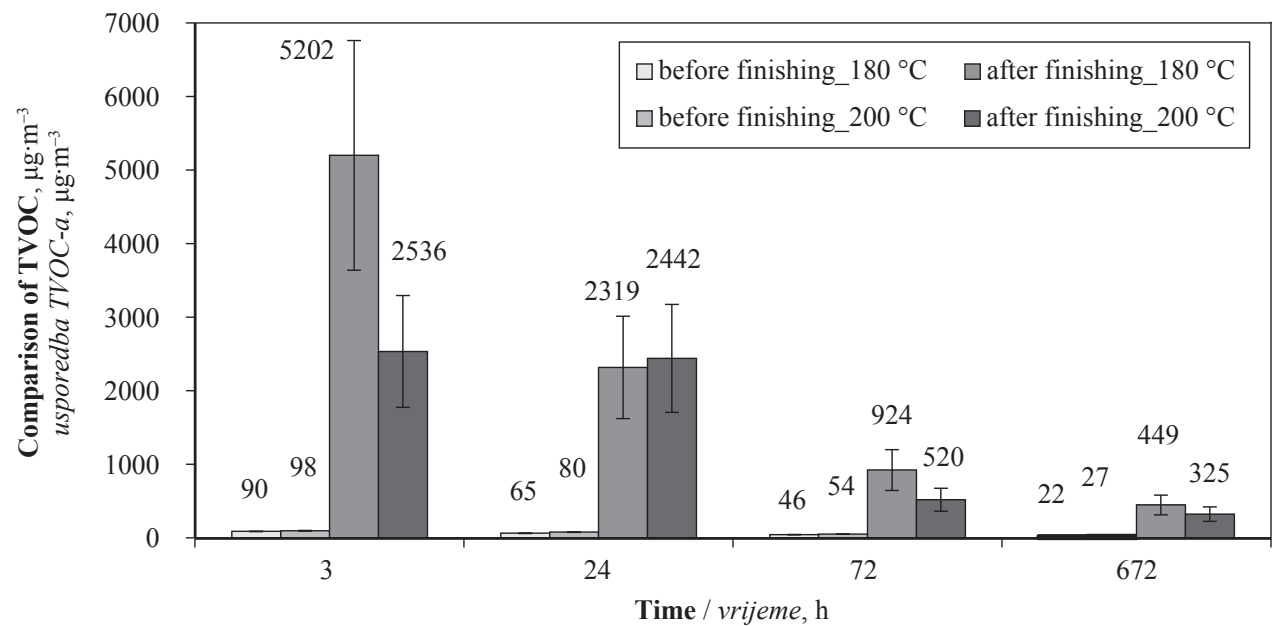

Figure 7 Comparison of TVOC from Norway Spruce - heat treated wood at $180{ }^{\circ} \mathrm{C}$ and $200{ }^{\circ} \mathrm{C}$, before and after finishing Slika 7. Usporedba TVOC-a koji emitira smrekovina - toplinski obrađena pri 180 i $200{ }^{\circ} \mathrm{C}$, prije i nakon završne obrade vodenim lakom

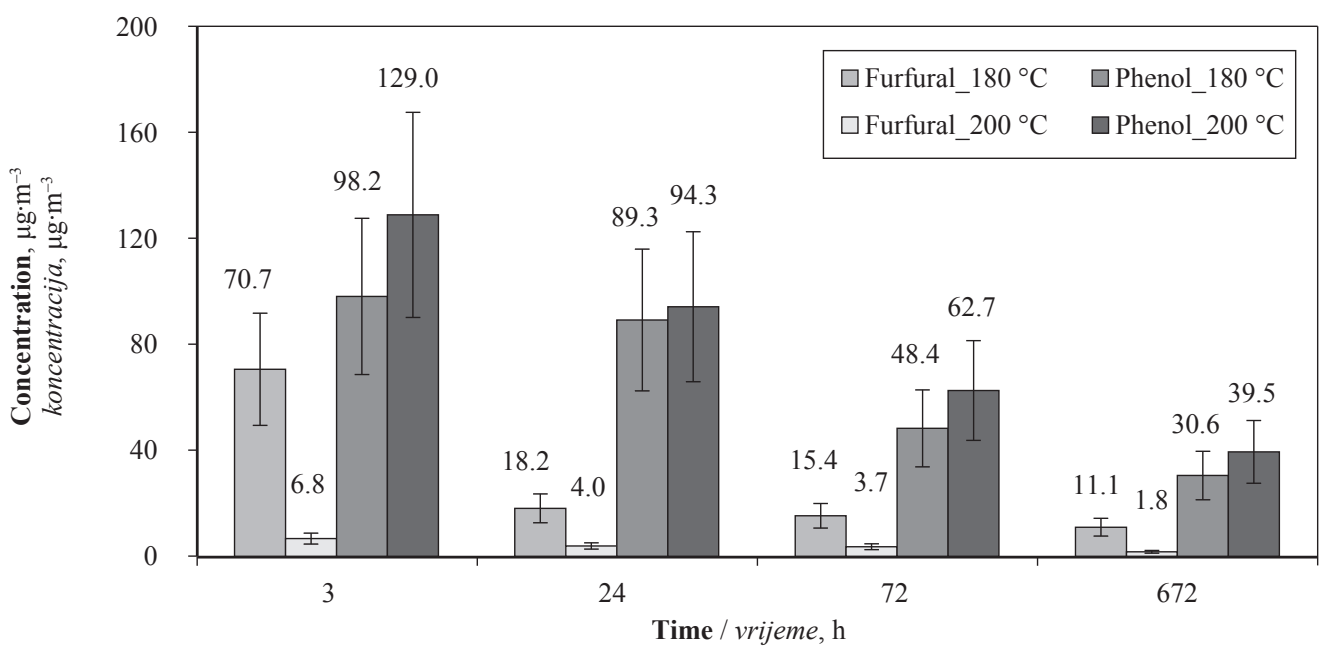

Figure 8 Amount of Furfural and Phenol emitted by heat-treated Poplar wood after finishing

Slika 8. Količina furfurala i fenola koju emitira toplinski obrađena topolovina nakon završne obrade vodenim lakom

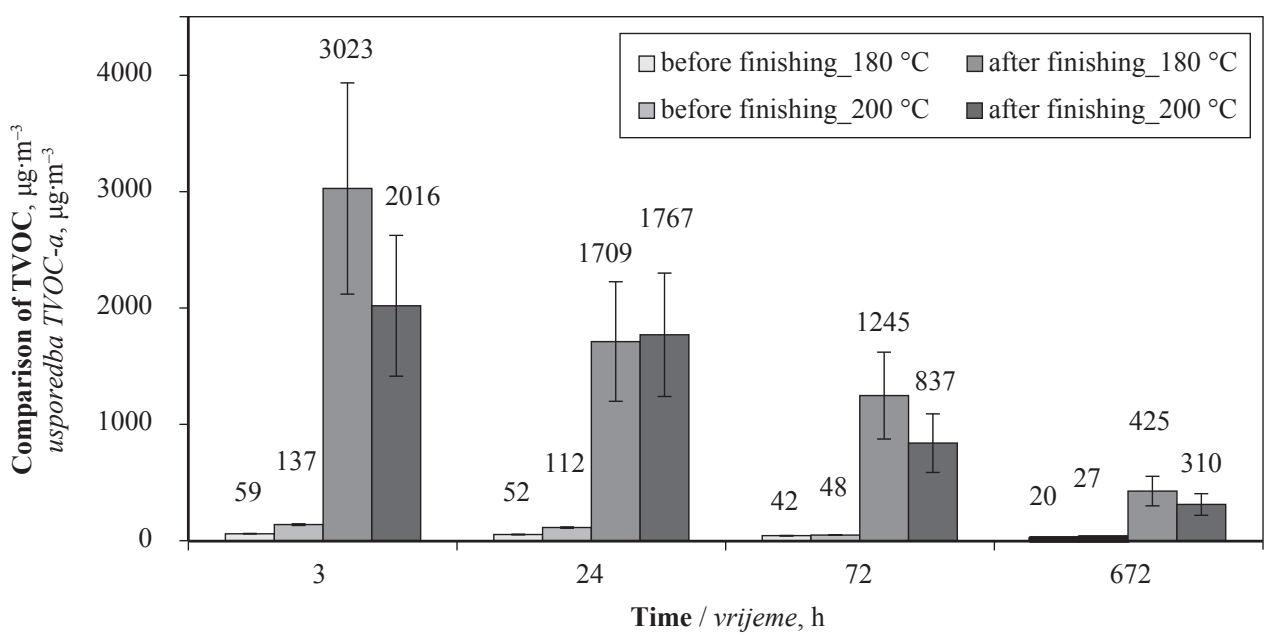

Figure 9 Comparison of TVOC from White Poplar - heat treated wood at $180{ }^{\circ} \mathrm{C}$ and $200{ }^{\circ} \mathrm{C}$, before and after finishing Slika 9. Usporedba TVOC-a koji emitira topolovina - toplinski obrađena pri 180 i $200^{\circ} \mathrm{C}$, prije i nakon završne obrade vodenim lakom 


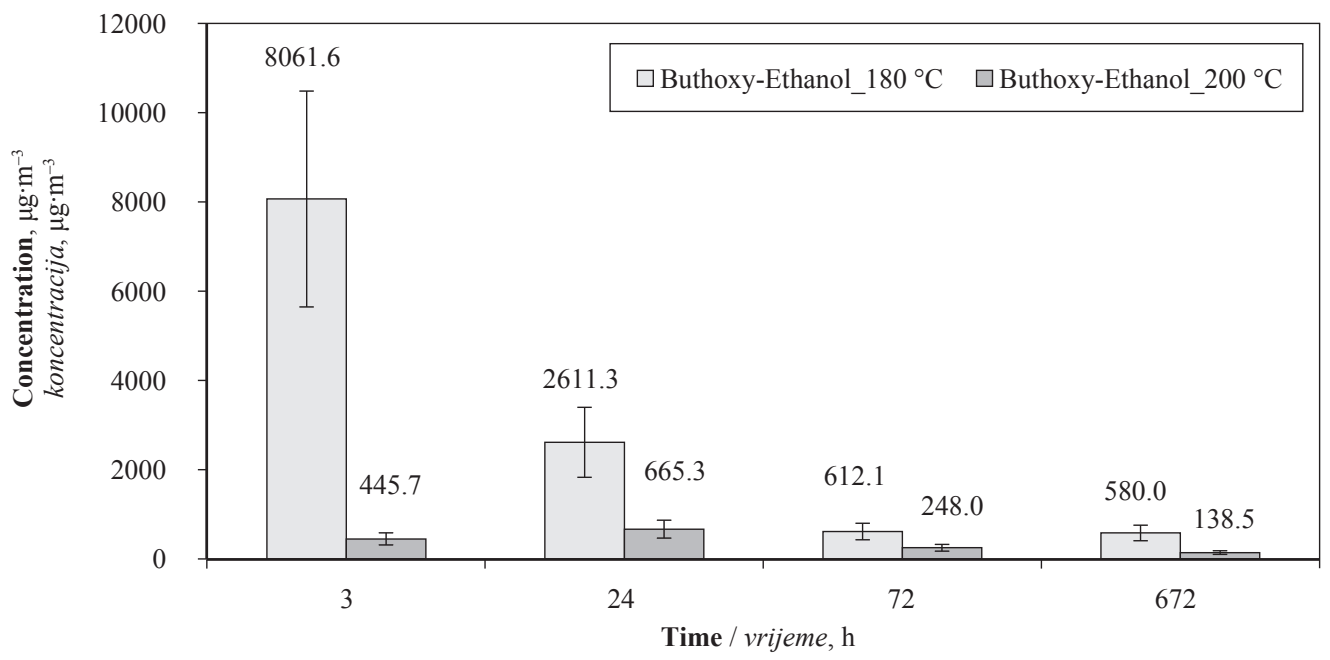

Figure 10 Amount of ButoxyEthanol emitted by heat-treated Spruce wood after finishing

Slika 10. Količina butoksietanola koji emitira toplinski obrađena smrekovina nakon završne obrade vodenim lakom

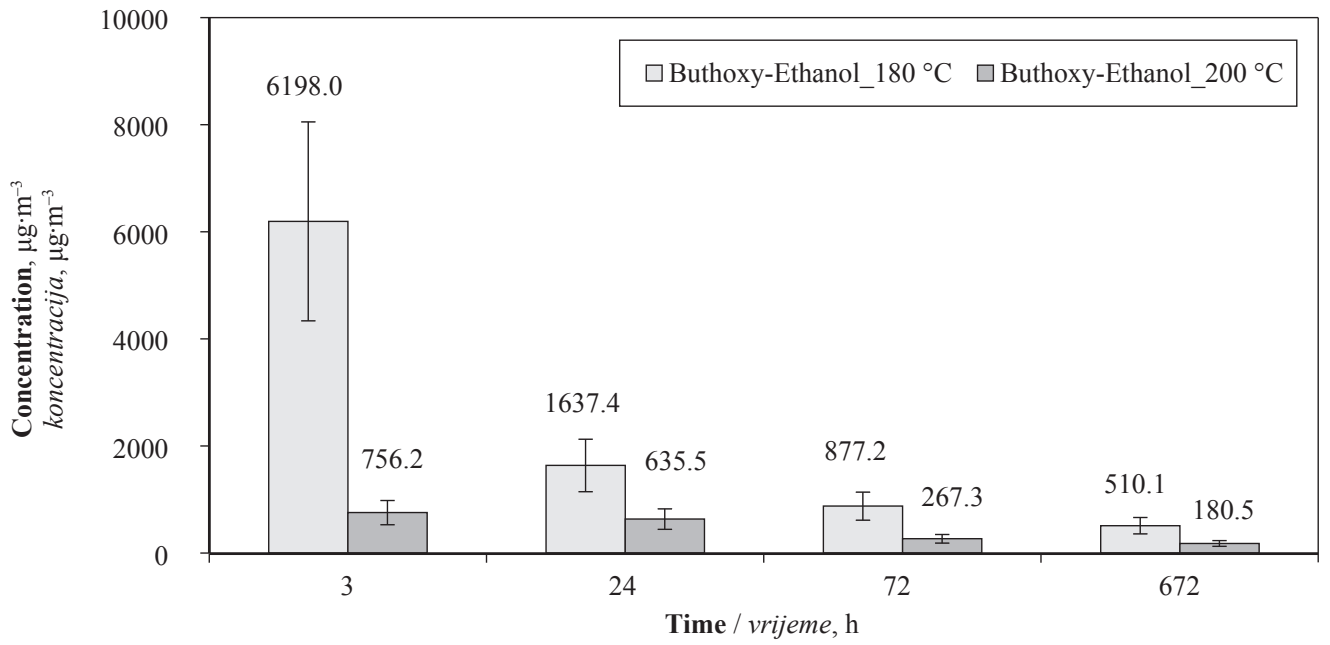

Figure 11 Amount of Buthoxy-Ethanol emitted by heat-treated Poplar wood after finishing

Slika 11. Količina butoksietanola koji emitira toplinski obrađena topolovina nakon završne obrade vodenim lakom

\section{Natural wood}

Tables 1 and 2 show the concentrations of VOCs emitted by natural, untreated, spruce and poplar wood. One of the major compounds found in our VOC collection from air-dried wood, hexanal, is known to be formed by oxidation of unsaturated fatty acids (Risholm-Sundman et al., 1998). Hexanal has also been a frequently analysed compound in the emissions of parquets and other wood products (Saarela, 1999).

Concentrations of other individual volatile organic compounds (VOCs) are very low and their values are comparable to background values (from 0.1 $\mu \mathrm{g} \cdot \mathrm{m}^{-3}$ for terpenes to almost $8 \mu \mathrm{g} \cdot \mathrm{m}^{-3}$ for furfural).

Values of TVOC (Total Volatile Organic compounds) are very low; however, values decrease depending on measurement time (Hyttinen et al., 2010).

\section{Heat treated wood before finishing}

The temperature of heat-treatment has a great influence on the amount of furfural emitted by tested heat-treated wood. The higher the temperature during spruce heat-treatment, the higher is the furfural emis- sion. However, during poplar heat treatment, the phenol emission is higher compared to furfural. Furfural and phenol are typical chemicals produced as a result of thermal degradation of wood components.

Furfural and 5-hydroxymethylfurfural (HMF) are common degradation products from monosugars, where HMF is formed from hexoses, whereas furfural mainly originates from pentoses (Fengel and Wegener, 1983, Alén et al., 2002; Peters et al., 2008). Heat treated process used for thermally modified wood emitted more VOC emissions at the temperature of $200{ }^{\circ} \mathrm{C}$ than at 180 ${ }^{\circ} \mathrm{C}$ (Manninen et al., 2002). The highest amount of Furfural emitted by heat treated spruce wood at $200{ }^{\circ} \mathrm{C}$ amounted to $227 \mu \mathrm{g} \cdot \mathrm{m}^{-3}$ after 3 hours, while the highest amount of Phenol emitted by heat treated poplar wood amounted to $185 \mu \mathrm{g} \cdot \mathrm{m}^{-3}$, measured at the same time. Heat treated wood (spruce and poplar) emitted a very small amount of terpenes (from 0.1 to $0.7 \mu \mathrm{g} \cdot \mathrm{m}^{-3}$ ).

\section{Heat treated wood after finishing}

The surface finished by waterborne lacquer does not decrease the amount of emissions escaping from 
heat-treated spruce and poplar wood. Finished heat treated wood emitted high amounts of Butoxy-ethanol, especially by heat treated process at $180{ }^{\circ} \mathrm{C}$ (spruce emitted more than $8000 \mu \mathrm{g} \cdot \mathrm{m}^{-3}$, poplar more than $\left.6000 \mu \mathrm{g} \cdot \mathrm{m}^{-3}\right)$. This effect has a significant impact on the value of TVOC.

TVOC emissions were significantly higher from heat-treated than from normal, air-dried wood samples. Terpenes were the main compounds emitted from softwood (spruce) and heat-treatment decreased their emissions. Terpenes partly evaporate and partly degrade during the heat-treatment process (McGraw et al., 1999). 4-Methyl-1-(1-methylethenyl)-benzene (pcymenene) and 1-methyl-2-(1-methylethyl)-benzene (o-cymene) were detected to a higher degree in airdried than in heat-treated softwood samples. These compounds are degradation products of camphene, delta-carene, and limonene (McGraw et al., 1999).

Emissions of aldehydes (furfural and hexanal) and carboxylic acids (acetic acid) were the most dominating compounds in heat treated softwood samples. In agreement with (Peters et al., 2008), emissions of furfural increased and those of hexanal decreased in heat-treated wood samples when compared to the air-dried ones. This phenomenon was observed in all wood species. This was to be expected, because hexanal belongs to the prevailing aldehydes in natural wood, whereas furfural is a major degradation product of hemicelluloses.

VOC emission profile changed dramatically during the heat treatment process. Although VOC emissions from the heat-treated samples were lower than from airtreated wood, oxidised organic compounds were also formed during the treatment. These have more unpleasant odour and are typically more irritating than terpenes, which dominate in the emissions of native softwoods. However, air-dried wood samples also emitted oxidised organic compounds, and terpenes might be oxidised to aldehydes and acids during the usage of the wood product (especially when ozone is preset in the air). In the present study, emissions of wood species were tested for a month. Actually, VOC emissions from wood (as any other materials) under constant conditions keep decreasing at least for one year. However, TVOC emissions from heat-treated wood products were relatively low already in the first days of testing.

\section{CONCLUSIONS}

5. ZAKLJUČAK

Based on the obtained results (Figures 2-11), it is concluded that the heat-treatment of wood increases the quantity of VOC emissions emitted by tested samples. The main difference was found in the amount of emitted furfural and phenol in the gaseous blend evaporated by heat-treated poplar under normal conditions.

The temperature of heat-treatment has a great influence on the amount of phenol and furfural emitted by tested heat-treated wood. Heat treated spruce emitted the highest amount of Furfural. The higher the temperature during the poplar heat-treatment, the higher is the phenol emission. Furfural and phenol are typical chemicals produced as a result of thermal degradation of wood components.
The surface finished by waterborne lacquer does not decrease the amount of emissions escaping from heat-treated spruce and poplar wood. Surprisingly, waterborne lacquers even elevated the amount of VOCs.

In the next step of this research, focus should be placed on the study of the influence of heat treatment on VOC emissions emitted by different kinds of wood and influence of finished surfaces of heat treated wood. It would also be important to find the correlation between the type of surface finishing and VOC emissions, i.e. to study the effect of the quality and quantity of VOC emissions emitted by heat treated wood on the way of finishing the heat-treated wood.

\section{Acknowledgement - Zahvala}

The author is grateful for the financial support of the Specific University Research Fund of the FFWT Mendel University in Brno for the project „IGA LDF VT_2018002, The covering materials used for upholstery furniture like source of VOC emission and odour in interior".

\section{REFERENCES}

\section{LITERATURA}

1. Alén, R.; Kotilainen, R.; Zaman, A., 2002: Thermochemical behavior of Norway spruce (Picea abies) at 180-225 ${ }^{\circ} \mathrm{C}$. Wood Science and Technology, 36 (2): 163-171. http://dx.doi.org/10.1007/s00226-001-0133-1.

2. Banerjee, S.; Su, W.; Wild, M. P.; Otwell, L. P.; Hittmeier, M. E.; Nichols, K. M., 1998: Wet line extension reduces VOCs from softwood drying. Environmental science \& technology, 32 (9): 1303-1307. http://dx.doi.org/10.1021/es970849o.

3. Čermák, P.; Rautkari, L.; Horáček, P.; Saake, B.; Rademacher, P.; Sablík, P., 2015: Analysis of Dimensional Stability of Thermally Modified Wood Affected by ReWetting Cycles. BioResources, 10 (2): 3242-3253. http://dx.doi.org/10.15376/biores.10.2.3242-3253.

4. Fengel, D.; Wegener, G. (eds.) 1983: Wood: chemistry, ultrastructure, reactions. Walter de Gruyter. https://books.google.cz/books?hl=cs\&lr=\&id=x1B4uIT Knt0C\&oi=fnd\&pg=PA1\&dq=Wood:+chemistry, + ultr astructure, + reactions. $+\&$ ots $=$ ece_45Qs1Z\&sig=HItPHZ KXVSnVQvgi3Ei-172jg6s\&redir_esc $=y \# v=$ onepage $\& q=$ Wood $\% 3 \mathrm{~A} \% 20$ chemistry $\% 2 \overline{\mathrm{C}} \% 20$ ultrastructure $\% 2 \mathrm{C} \% 20$ reactions. \&f $=$ false.

5. Hill, C. A., 2007: Wood modification: chemical, thermal and other processes (Vol. 5). John Wiley \& Sons. http://dx.doi.org/10.1002/0470021748.

6. Hyttinen, M.; Masalin-Weijo, M.; Kalliokoski, P.; Pasanen, P., 2010: Comparison of VOC emissions between airdried and heat-treated Norway spruce (Picea abies), Scots pine (Pinus sylvesteris) and European aspen (Populus tremula) wood. Atmospheric Environment, 44 (38): 50285033. http://dx.doi.org/10.1016/j.atmosenv.2010.07.018.

7. Kamdem, D. P.; Pizzi, A.; Triboulot, M. C., 2000: Heattreated timber: potentially toxic byproducts presence and extent of wood cell wall degradation. European Journal of Wood and Wood Products, 58 (4): 253-257. http://dx.doi.org/10.1007/s001070050420.

8. Kamdem, D. P.; Pizzi, A.; Jermannaud, A., 2002: Durability of heat-treated wood. European Journal of Wood and Wood Products, 60 (1): 1-6. 
http://dx.doi.org/10.1007/s00107-001-0261-1.

9. Kotilainen, R., 2000. Chemical changes in wood during heating at $150-260{ }^{\circ} \mathrm{C}$. PhD Thesis, Jyvaskyla University. Research report 80, Finland. https://books.google. cz/books/about/Chemical_changes_in_wood_during_ heating.html?id=EF-djwEACAAJ\&redir_esc $=$ y.

10. Lavery, M. R.; Milota, M. R., 2000: VOC emissions from Douglas-fir: Comparing a commercial and a laboratory kiln. Forest Products Journal, 50 (7/8): 39. http://search. proquest.com/docview/214640257?pq-origsite=gscholar.

11. Long, L.; Wang, J. L., 2007: Aldehyde and terpene emissions from four species of wood at normal temperature. China Wood Industry, 21 (3): 14-17. http://dx.doi.org/10.15376/biores.11.2.3550-3560.

12. Manninen, A. M.; Pasanen, P.; Holopainen, J. K., 2002: Comparing the VOC emissions between air-dried and heat-treated Scots pine wood. Atmospheric Environment, 36 (11): 1763-1768.

http://dx.doi.org/10.1016/S1352-2310(02)00152-8.

13. McGraw, G. W.; Hemingway, R. W.; Ingram Jr., L. L.; Canady, C. S.; McGraw, W. B., 1999. Thermal degradation of terpenes: camphene, $\Delta^{3}$-carene, limonene, and aterpinene. Environmental Science and Technology 33: 4029-4033. http://dx.doi.org/10.1021/es9810641.

14. Militz, H., 2002. Thermal treatment of wood: European processes and their background. In: 33rd Annual Meeting, 12 - 17 May, Cardiff-Wales, IRG/WP 02-40241, 4, pp. 1-17. http://www.irg-wp.com/irgdocs/details.php? f6f6ffad-b3c3-433d-aaaa-647a154fd4c7.

15. Miller, D. D., 1998: Food chemistry - A laboratory manual, Wiley-Interscience, John Wiley \& Sons, New York, Chichester, Weinhiem, Brisbane, Singapore, Toronto.

16. Milota, M. R., 2000: Emissions from wood drying: the science and the issues. Forest Products Journal, 50 (6): 10. http://search.proquest.com/docview/214640755?pqorigsite $=$ gscholar.

17. Peters, J.; Fischer, K.; Fischer, S., 2008: Characterization of emissions of thermally modified wood and their reduction by chemical treatment. BioResources, 3 (2): 491-502. https://ojs.cnr.ncsu.edu/index.php/BioRes/article/view/BioRes_03_2_0491_Peters_FF_Emissions_Thermal_Wood.

18. Sandermann, W.; Augustin, $\bar{H}_{\text {., }}$ 1964: Chemische Untersuchungen über die thermische Zersetzung von HolzDritte Mitteilung: Chemische Untersuchung des Zersetzungsablaufs. European Journal of Wood and Wood Products, 22 (10): 377-386. http://dx.doi.org/10.1007/BF02628346.

19. Risholm-Sundman, M.; Lundgren, M.; Vestin, E.; Herder, P., 1998: Emissions of acetic acid and other volatile organic compounds from different species of solid wood. Holz als Roh-und Werkstoff, 56: 125-129. https://link. springer.com/content/pdf/10.1007/s001070050282.pdf.

20. Saarela, K., 1999: Emission from floor coverings. In: Salthammer, T. (ed.). Organic Indoor Air Pollutants. Occurrence, Measurement, Evaluation. Wiley-VCH, Weinheim, pp. 185-202. https://doi.org/10.1002/9783527613663.ch14.

21. Sjöström, E., 1993: Wood chemistry. Fundamentals and Applications, second ed. Academic Press, Inc., San Diego, pp. 1-293. https://books.google.cz/books?hl=cs\&lr=

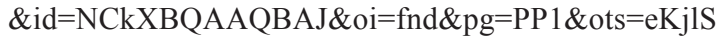
UuE22\&sig=uj1m8SzUutePmlVnpVV3hEgkA0Y\&red ir_esc $=\mathrm{y} \# \mathrm{v}=$ onepage \&q\&f$=$ false.

22. Sivonen, H.; Maunu, S. L.; Sundholm, F.; Jämsä, S.; Viitaniemi, P., 2002: Magnetic resonance studies on thermally modified wood. Holzforschung, 56: 648-654. https://www.degruyter.com/view/j/hfsg.2002.56.issue-6/ hf.2002.098/hf.2002.098.xml.
23. Syrjanen, T.; Oy, K., 2001: Production and classification of heat treated wood in Finland, Review on heat treatments of wood. In: Proceedings of the special seminar held in Antibes, France. http://projects.bre.co.uk/ecotan/ pdf/Heat_treatment_processes_Andreas_Rapp\%20.pdf.

24. Su, W.; Boerner, J. R.; Hooda, U.; Yan, H.; Banerjee, S.; Shmulsky, R.; Conners, T. E., 1999: VOC extraction from softwood through low-headspace heating. Holzforschung, 53 (6): 641-647. http://dx.doi.org/10.1515/HF.1999.105.

25. Tjeerdsma, B. F.; Militz, H., 2005: Chemical changes in hydrothermal treated wood: FTIR analysis of combined hydrothermal and dry heat-treated wood. Holz als rohund Werkstoff, 63 (2): 102-111. http://dx.doi.org/10.1007/s00107-004-0532-8.

26. Westin, M.; Simonson, R.; Östman, B., 2001: Kraft lignin wood fiberboards - The effect of kraft lignin addition to wood chips or board pulp prior to fiberboard production. European Journal of Wood and Wood Products, 58 (6): 393-400. http://dx.doi.org/10.1007/s001070050451.

27. Windeisen, E.; Strobel, C.; Wegener, G., 2007: Chemical changes during the production of thermo-treated beech wood. Wood Science and Technology, 41 (6): 523-536. http://dx.doi.org/10.1007/s00226-007-0146-5.

28. Windeisen, E.; Wegener, G., 2009: Chemical characterization and comparison of thermally treated beech and ash wood. In Materials Science Forum, Vol. 599, pp. 143158, Trans Tech Publications.

http://dx.doi.org/10.4028/www.scientific.net/MSF.599.143.

29. Wu, J.; Milota, M. R., 1999: Effect of temperature and humidity on total hydrocarbon emissions from Douglasfir lumber. Forest products journal, 49 (6): 52. http:// search.proquest.com/docview/214624706?pq-origsite= gscholar.

30. ***ISO International Standardisation Organisation 2007: ISO 16000-1. General aspects of sampling strategy. Geneva: International Organization for Standardization.

31. ***ISO International Standardisation Organisation 2007: ISO 16000-5. Sampling strategy for volatile organic compounds (VOCs). Geneva: International Organization for Standardization.

32. ***ISO International Standardisation Organisation 2007 : ISO 16000-6. Determination of volatile organic compounds indoor and test chamber air by active sampling on Tenax $\mathrm{TA}^{\circledR}$ sorbent, thermal desorption and chromatography using MS/FID. Geneva: International Organization for Standardization.

33. ***ISO International Standardisation Organisation 2007: ISO 16000-9. Determination of emissions of volatile organic compounds from building materials and furniture - test chamber method. Geneva: International Organization for Standardization.

\section{Corresponding address:}

\section{Ing. PETR ČECH, Ph.D.}

Department of Furniture, Design and Habitation Faculty of Forestry and Wood Technology Mendel University in Brno - Zemědělská 3, Brno, 613 00, CZECH REPUBLIC e-mail: Cech.P007@seznam.cz 